\title{
Predicción de fracaso de las puntas nasales de alto flujo con la escala HACOR, en la insuficiencia respiratoria aguda de pacientes con SARS-CoV-2
}

Prediction of failure of the high-flow nasal tips with the HACOR scale, in acute respiratory insufficiency in patients with SARS-CoV-2 Previsão de falha das pontas nasais de alto fluxo com a escala HACOR, na insuficiência respiratória aguda de pacientes com SARS-CoV-2

Ibzan Jahzeel Salvador-Ibarra, ${ }^{*}$ Alejandro Pizaña Dávila*

\section{RESUMEN}

La insuficiencia respiratoria hipoxémica aguda (IRHA) es una de las principales causas de ingreso en la Unidad de Terapia Intensiva en los pacientes adultos, alrededor de $40 \%$ de estos pacientes ameritan intubación endotraqueal. La escala HACOR fue diseñada y validada como una puntuación de predicción clínica para el fracaso de la ventilación no invasiva (VNI) en pacientes con IRHA de diferentes etiologías.

Objetivo: Analizar la capacidad predictiva de la escala HACOR en relación a fracaso de las puntas nasales de alto flujo (PNAF).

Material y métodos: Se realizó un estudio retrospectivo, observacional en la Terapia Intensiva del Hospital Ángeles Mocel, las PNAF se iniciaron cuando e paciente presentaba IRHA, se calculó la escala HACOR a la hora y a las dos horas de colocación de PNAF, la capacidad de predecir el fracaso de las PNAF de la escala HACOR se determinó calculando el área bajo la curva de «característica operativa del receptor» (ROC).

Resultados: La escala HACOR con mayor poder discriminatorio se obtuvo a las dos horas de inicio de las PNAF con un punto de corte de 5 puntos con un área bajo la curva (AUC): 0.82 (IC 95\% 0.67-0.98), se obtuvo una sensibilidad 84\% IC 95\% (56.6-89.9\%), especificidad 81\% IC 95\% (40.9-92.9\%).

Conclusiones: Un puntaje mayor o igual a 5 puntos en la escala HACOR predice fracaso de las PNAF y mayor mortalidad con una sensibilidad y especificidad mayor de $80 \%$ en los pacientes con neumonía por COVID-19.

Palabras clave: Insuficiencia respiratoria aguda, puntas nasales de alto flujo, escala HACOR, éxito, fracaso, ventilación mecánica.

\section{ABSTRACT}

Acute hypoxemic respiratory failure (AHRF) is one of the main causes of admission to the intensive care unit in adult patients, around $40 \%$ of these patients require endotracheal intubation. The HACOR scale was designed and validated as a clinical prediction score for non-invasive ventilation (NIV) failure in patients with HAl of different etiologies.

Objective: To analyze the predictive capacity of the HACOR scale in relation to the failure of the PNAF.

Material and methods: A retrospective, observational study was carried out in the Intensive Care Unit at Hospital Ángeles Mocel, the PNAF was started when the patient had AHRF, the HACOR scale was calculated at one hour and 2 hours after the placement of the PNAF, the ability to predict HACOR scale PNAF failure was determined by calculating the area under the "receiver operating characteristic" (ROC) curve.

Results: The HACOR scale with the highest discriminatory power was obtained 2 hours after the start of the PNAF with a cut-off point of 5 points with an area under the curve (AUC): 0.82 (CI 95\% 0.67-0.98), it was obtained a sensitivity $84 \%$ Cl 95\% (56.6-89.9\%), specificity 81\% Cl 95\% (40.9-92.9\%).

Conclusions: A score greater than or equal to 5 points on the HACOR scale predicts failure of the PNAF and higher mortality with a sensitivity and specificity greater than $80 \%$ in patients with COVID-19 pneumonia.

Keywords: Acute respiratory failure, high-flow nasal tips, HACOR scale, success, failure, mechanical ventilation.

\section{* Hospital Ángeles Mocel. Ciudad de México, México.}

Recibido: 15/12/2020. Aceptado: 09/02/2021.

Citar como: Salvador-Ibarra IJ, Pizaña DA. Predicción de fracaso de las puntas nasales de alto flujo con la escala HACOR, en la insuficiencia respiratoria aguda de pacientes con SARS-CoV-2. Med Crit. 2021;35(5):250-255. https://dx.doi.org/10.35366/102353

www.medigraphic.com/medicinacritica

\section{RESUMO}

A insuficiência respiratória hipoxêmica aguda (IRHA) é uma das principais causas de admissão em unidade de Terapia Intensiva em Pacientes Adultos, cerca de $40 \%$ desses pacientes requerem intubação endotraqueal. A escala HACOR foi desenhada e validada como um escore de predição clínica para falha da ventilação não invasiva (VNI) em pacientes com IRHA de diferentes etiologias.

Objetivo: Analisar a capacidade preditiva da escala HACOR em relação à falha das PNAF.

Material e métodos: Foi realizado um estudo retrospectivo e observacional na Unidade de Terapia Intensiva do Hospital Ángeles Mocel, a PNAF foi iniciada quando o paciente tinha IRHA, a escala HACOR foi calculada uma hora e 2 horas após a colocação da PNAF, a capacidade de previsão do fracasso das PNAF da escala HACOR foi determinada pelo cálculo da área sob a curva «receiver operating characteristics» (ROC).

Resultados: A escala HACOR com maior poder discriminatório foi obtida 2 horas após o início das PNAF com ponto de corte de 5 pontos com área sob a curva (AUC): 0.82 (IC 95\% 0.67-0.98), foi obtida uma sensibilidade de $84 \%$ IC 95\% (56.6\%-89.9\%), especificidade $81 \%$ IC 95\% (40.9\%-92.9\%).

Conclusões: Uma pontuação maior ou igual a 5 pontos na escala HACOR prediz falha das PNAF e maior mortalidade com sensibilidade e especificidade maior à $80 \%$ em pacientes com pneumonia COVID-19.

Palavras-chave: Insuficiência respiratória aguda, pontas nasais de alto fluxo, escala HACOR, êxito, falha, ventilação mecânica.

\section{INTRODUCCIÓN}

La insuficiencia respiratoria hipoxémica aguda (IRHA) es una de las principales causas de ingreso en la Unidad de Terapia Intensiva en los pacientes adultos, ${ }^{1}$ alrededor de $40 \%$ de estos pacientes ameritan intubación endotraqueal. ${ }^{2}$ Respecto a la enfermedad infecciosa causada por el SARS-CoV-2, 3.4\% de los pacientes infectados en China presentaron un síndrome de insuficiencia respiratoria aguda (SIRA), lo que indica que $40.3 \%$ de los pacientes presentaron una patología grave. Los resultados publicados en el análisis de las características clínicas en una cohorte seleccionada de 1,099 enfermos de COVID-19 en toda China mostraron que $24.8 \%$ necesitaron ingresar a las unidades de terapia intensiva (UTI), uso de ventilación mecánica, tanto invasiva como no invasiva, o fallecieron. ${ }^{3}$

La oxigenoterapia es el tratamiento de primera línea en pacientes hipoxémicos. El oxígeno se puede administrar mediante dispositivos de bajo flujo (hasta 15 L/ min) como cánulas nasales, mascarilla y máscaras con bolsa reservorio. La fracción de oxígeno inspirado $\left(\mathrm{FiO}_{2}\right)$ obtenida con estos dispositivos varía con el flujo de oxígeno y con el flujo inspiratorio máximo del pa- 
ciente, el sistema de administración y las características del dispositivo. ${ }^{4}$ Los dispositivos de administración convencionales tienen varios inconvenientes además de la limitación de la $\mathrm{FiO}_{2}$ que restringe su eficacia y tolerancia, como una humidificación insuficiente y un calentamiento insuficiente del gas inspirado a flujos altos que provocan malestar en el paciente. ${ }^{5}$ Las puntas nasales de alto flujo (PNAF) son un soporte respiratorio no invasivo diseñado para administrar de 30 a $60 \mathrm{~L} / \mathrm{min}$ de una mezcla de aire y oxígeno, calentada y humidificada a través de puntas nasales diseñadas específicamente. Las PNAF permiten la modificación de dos configuraciones principales: el porcentaje de oxígeno suministrado y la tasa de flujo de gas. El flujo nasal puede suministrar una mezcla de aire y oxígeno con una fracción de oxígeno inspirada $\left(\mathrm{FiO}_{2}\right)$ que oscilan entre 0.21 y $1.0 .{ }^{6}$

Hay un uso cada vez mayor de la oxigenoterapia con PNAF para la IRHA, alentado por la evidencia que sugiere tasas de intubación reducidas y posiblemente una mortalidad más baja. ${ }^{7}$ Ofrece ventajas reportadas respecto a la comodidad del paciente, mejor oxigenación y disminución del trabajo respiratorio, puntuaciones de disnea más bajas y sequedad bucal reducida. ${ }^{8}$

La ventilación mecánica invasiva (VMI) se asocia con eventos adversos como neumonía asociada al ventilador, lesión pulmonar inducida por el ventilador y el potencial de sedación excesiva. ${ }^{9,10}$ Existe la necesidad de mejorar la predicción temprana de falla de PNAF, ya que la intubación tardía se asocia con un aumento de la mortalidad. ${ }^{11}$

Las PNAF han sido utilizadas en toda una gama de escenarios clínicos que cursan con IRHA, tales como: EAP (edema agudo de pulmonar), EPOC (enfermedad pulmonar obstructiva crónica), postoperatorio de cirugía cardiovascular, tórax, abdomen, trasplante de pulmón, procedimientos como la oxigenación antes de la intubación orotraqueal (IOT), broncoscopias y ante la pandemia por SAR-CoV-2 su uso se ha generalizado.

La escala HACOR fue diseñada y validada como una puntuación de predicción clínica para el fracaso de la ventilación no invasiva (VNI) en pacientes con IRHA de diferentes etiologías. La escala HACOR se basa en varias variables objetivas fácilmente medibles evaluadas en el momento del inicio de la VNI: frecuencia cardiaca, valor de $\mathrm{pH}$ arterial, nivel de conciencia mediante la escala de coma de Glasgow, oxigenación mediante la relación $\mathrm{PaO}_{2} / \mathrm{FiO}_{2}$ y frecuencia respiratoria (Tabla 1). ${ }^{12}$ Decidimos realizar una modificación mínima a la escala sustituyendo la $\mathrm{PaO}_{2} / \mathrm{FiO}_{2}$ por el índice $\mathrm{SpO}_{2} / \mathrm{FiO}_{2}$ para facilitar su aplicación.

El objetivo principal de este estudio fue analizar la capacidad predictiva de la escala HACOR en relación al fracaso de las PNAF, medida a la hora y a las dos horas de colocación del dispositivo de alto en pacientes con SIRA secundario a COVID-19.
Los objetivos secundarios fueron identificar los datos demográficos de los pacientes que ingresaron con insuficiencia respiratoria, relacionar los cambios en las variables fisiológicas medidas a la hora y a las dos horas de ventilación con PNAF, relacionar la tolerancia o el fracaso de ventilación con PNAF con la mortalidad.

\section{MATERIAL Y MÉTODOS}

Se realizó un estudio retrospectivo, observacional en la Unidad de Terapia Intensiva del Hospital Ángeles Mocel destinada a la atención de pacientes con diagnóstico de COVID-19 durante el periodo comprendido del 1 de marzo de 2020 al 30 de junio de 2020. El estudio ha sido aprobado por el comité ético de la institución. Criterios de inclusión: pacientes mayores de 18 años de edad que presentaron IRHA y fueron tratados con puntas nasales de alto flujo con prueba de reacción en cadena de la polimerasa (PCR por sus siglas en inglés) para SARS-CoV-2 por hisopado nasofaríngeo positiva. Los criterios de exclusión fueron: aquellos pacientes con requerimiento de intubación urgente, presencia de agitación severa y poca tolerancia de las PNAF, inestabilidad cardiovascular sin respuesta a la administración de líquidos parenterales, fármacos vasoactivos y pacientes trasladados de otra unidad hospitalaria que ya contaban con manejo avanzado de la vía aérea.

Las PNAF se iniciaron cuando el paciente presentaba IRHA definida como la presencia de disnea moderada a grave, frecuencia respiratoria igual o superior

Tabla 1: Escala HACOR para predicción temprana de fallo de las puntas nasales de alto flujo.

\begin{tabular}{lcc}
\hline & Categoría & Puntos \\
\hline $\mathrm{FC}$ & $\leq 120$ & 0 \\
& $>120$ & 1 \\
$\mathrm{pH}$ & $\geq 7.35$ & 0 \\
& $7.30-7.34$ & 2 \\
& $7.25-7.29$ & 3 \\
$\mathrm{ECG}$ & $<7.25$ & 4 \\
& 15 & 0 \\
& $13-14$ & 2 \\
$\mathrm{SpO}_{2} / \mathrm{FiO}_{2}$ & $11-12$ & 5 \\
& $\leq 10$ & 10 \\
& $>235$ & 0 \\
& $211-235$ & 2 \\
& $187-210$ & 3 \\
$\mathrm{FR}$ & $163-186$ & 4 \\
& $139-162$ & 5 \\
& $\leq 139$ & 6 \\
& $\leq 30$ & 0 \\
& $31-35$ & 1 \\
& $36-40$ & 2 \\
& $41-45$ & 3 \\
& $>45$ & 4 \\
\hline
\end{tabular}

$\mathrm{FC}=$ frecuencia cardiaca; $\mathrm{ECG}$ = escala de coma de Glasgow; $\mathrm{SpO}_{2}=$ saturación capilar de oxígeno; $\mathrm{FiO}_{2}$ = fracción inspirada de oxígeno; $\mathrm{FR}$ = frecuencia respiratoria.

Puntaje mínimo: 0 puntos, puntaje máximo: 25 puntos. 
Tabla 2: Variables generales de la muestra.

\begin{tabular}{lc}
\hline Variables & Media $\pm \mathrm{DE}$ \\
\hline Género, $\mathrm{n}(\%)$ & $21(70.0)$ \\
Masculino & $9(30.0)$ \\
Femenino & $56 \pm 18.52$ \\
Edad (años) & $28 \pm 4.8$ \\
IMC & $32.5 \pm 10.2$ \\
HDL & $77.17 \pm 15.7$ \\
Peso & $1.64 \pm 0.10$ \\
Talla & $11.8 \pm 9.9$ \\
SOFA & $11.3 \pm 9.3$ \\
APACHE & $19(63.3)$ \\
Defunciones, $n(\%)$ & \\
\hline
\end{tabular}

IMC = índice de masa corporal; $\mathrm{HDL}=$ lipoproteínas de alta densidad; SOFA = escala de evaluación de falla orgánica secuencial; APACHE = Physiology and Chronic Health disease Classification System.

a 25 respiraciones por minuto, utilización activa de la musculatura respiratoria accesoria o movimiento abdominal paradójico y presencia de hipoxemia, definida la presencia de una $\mathrm{SpO}_{2} / \mathrm{FiO}_{2}<235$ con oxígeno suplementario. Todos los pacientes fueron monitorizados de forma continua con electrocardiograma, pulsioximetría, tensión arterial y frecuencia respiratoria. Se calculó la escala HACOR a la hora y a las dos horas de colocación del dispositivo de PNAF. Para administrar el sistema de PNAF se utilizaron tres tipos de dispositivos AIRVO 2 (Fisher \& Paykel Healthcare, Auckland, Nueva Zelanda), Air-Oxygen Blender, USA y MaxVenturi ${ }^{\circledR}$, oxygen sensor \& Hewitt clamp 0-120 L/min (High-Flow) con Optiflow (MR850 calentador y humidificador, con tubo RT202, Precision Medical 5300).

El éxito de las PNAF se definió al evitar la intubación endotraqueal, consciente durante al menos 24 horas sin signos de insuficiencia respiratoria y egreso de la UTI. Se consideró fracaso de las PNAF cuando se requirió de intubación y soporte con VMI con base en los siguientes criterios: paro cardiaco o respiratorio, falla para mantener $\mathrm{SpO}_{2} / \mathrm{FiO}_{2}>150 \mathrm{mmHg}$, Glasgow menor o igual a 8 puntos, deterioro en las mediciones de intercambio gaseoso, empeoramiento de los signos y síntomas de insuficiencia respiratoria, $\mathrm{SpO}_{2}$ menor de $88 \%$, FR mayor de $25 \mathrm{rpm}$. Se realizó análisis estadístico para las variables cuantitativas con una distribución paramétrica y fueron presentadas como medias y desviación estándar, y para aquellas con distribución no paramétrica como medianas, mínimos y máximos, dividimos la muestra en dos grupos, el grupo 1 representa a los pacientes que fallecieron y el grupo 2 a los que sobrevivieron y los comparamos utilizando una prueba exacta de Fisher para datos categóricos con distribución no paramétrica, representados con números y porcentajes. Además utilizamos una prueba de Mann-Whitney para comparar medianas de variables con distribución no paramétrica. Se utilizó una prueba de Wilcoxon para datos emparejados para comparar las variables de la escala HACOR a la hora vs a las dos horas, con la intención de identificar alguna variable que tuviera mayores cambios significativos, un valor $p<0.05$ se consideró significativo. El riesgo relativo (RR) se calculó con un intervalo de confianza del $95 \%$. La capacidad de predecir el fracaso de las PNAF de la escala HACOR se determinó calculando el área bajo la curva de "característica operativa del receptor» (ROC), calculando la sensibilidad, especificidad e índice de probabilidad para el punto de corte óptimo de la escala. Los análisis se realizaron a través del programa Statistical Package for the Social Sciences (SPSS) 26.0 (IBM, Armonk, NY).

\section{RESULTADOS}

Durante el periodo de estudio ingresaron en la UTI un total de 30 pacientes tratados con PNAF, se recogieron variables demográficas y clínicas al ingreso, se calculó un IMC en todos los pacientes. Observamos que $70 \%$ de los pacientes eran del género masculino (21) con una media de edad de $56 \pm 18.52$ años, el peso fue de $77.17 \pm 15.7$ y la talla de $1.64 \pm 0.10$. Identificamos un $63.3 \%$ de pacientes fallecidos (19). El grado de gravedad de la enfermedad se calculó utilizando el Acute Physiology and Chronic Health disease Classification System II (APACHE II), calculado en las primeras 24 horas de estancia en la UTI, y el grado de disfunción multiorgánica utilizando la escala de evaluación de falla orgánica secuencial (SOFA) con una media de $11.8 \pm$ 9.9, APACHE de $11.3 \pm 9.3$ (Tabla 2). Al comparar los dos grupos (fracaso vs éxito de las PNAF) identificamos diferencias significativas para la puntuación de SOFA con una $p=0.049$ (Tabla 3 y Figura 1). Los pacientes con fracaso de VNI presentaron valores de la escala HACOR más altos que los que tuvieron éxito. La capacidad para predecir el fracaso de las PNAF utilizando la escala HACOR se obtuvo mediante curva de ROC (características operativas del receptor) el momento de medición de la escala HACOR con mayor poder discriminatorio se obtuvo a las dos horas de inicio de las PNAF con un punto de corte de 5 puntos con un área bajo la curva $(A \cup C)=0.82$ (IC 95\% 0.67-0.98) (Figura 2), se obtuvo una sensibilidad $84 \%$ IC $95 \%$ (56.6$89.9 \%$ ), especificidad $81 \%$ IC $95 \%$ (40.9-92.9\%) con un valor predictivo positivo $89.5 \%$, valor predictivo negativo $54.5 \%$ y riesgo relativo 10.20 . En la prueba de Wilcoxon para datos emparejados, identificamos que la frecuencia respiratoria y la escala coma de Glasgow presentaron el mayor cambio a las dos horas con una $p$ $=0.036$ y 0.033 respectivamente (Tabla 4). De acuerdo con este resultado, al no observar cambios significativos en el pH de la gasometría, se realizó una curva ROC con un puntaje HACOR sin incluir el $\mathrm{pH}$, ya que de nuestra muestra sólo un paciente desarrolló acidemia a 
las dos horas de la ventilación con PNAF y se encontró una $A U C=0.82$ (IC 95\% 0.66-0.98) sensibilidad $84 \%$ y especificidad $81 \%$.

\section{DISCUSIÓN}

En los últimos años, el flujo alto de oxígeno por vía nasal ha ganado una popularidad importante entre los intensivistas para el manejo de pacientes con insuficiencia respiratoria aguda, llenando un vacío en la escalada del soporte ventilatorio entre el oxígeno de la mascarilla y la ventilación mecánica no invasiva o invasiva. ${ }^{13} \mathrm{Se}$ ha observado que las PNAF reducen significativamente la frecuencia respiratoria, frecuencia cardiaca, puntuación de disnea, retracción supraclavicular, asincronía toracoabdominal y aumenta la oximetría de pulso. ${ }^{14}$ El estudio FLORALI mostró en un subgrupo post hoc

Tabla 3: Diferencias de las variables entre los pacientes con fracaso y éxito a las puntas nasales de alto flujo.

\begin{tabular}{lccc}
\hline Variables & $\begin{array}{c}\text { Con fracaso de PNAF } \\
\mathrm{N}=19(63.3 \%)\end{array}$ & $\begin{array}{c}\text { Éxito de PNAF } \\
\mathrm{N}=11(36.7 \%)\end{array}$ & $\mathrm{p}$ \\
\hline Género, $\mathrm{n}(\%)$ & & & \\
$\quad$ Masculino & $13(68.4)$ & $8(72.7)$ & 0.571 \\
$\quad$ Femenino & $6(31.5)$ & $3(27.2)$ & 0.622 \\
IMC & $28.7(17.2-35.5)$ & $28.01(20.5-35.9)$ & 1.000 \\
HDL & $35.1(14.8-45.5)$ & $34.9(5-47)$ & 1.000 \\
Peso & $80(43-115)$ & $74(55-104)$ & 0.466 \\
Talla & $1.65(1.48-1.86)$ & $1.70(1.4-1.8)$ & 0.450 \\
SOFA & $6.4(5.4-50)$ & $6.4(0-21.5)$ & 0.049 \\
APACHE & $8(4-55)$ & $8(4-15)$ & 0.442 \\
\hline
\end{tabular}

PNAF = puntas nasales de alto flujo; $I M C=$ índice de masa corporal; $\mathrm{HDL}=$ lipoproteínas de alta densidad; SOFA = escala de evaluación de falla orgánica secuencial; APACHE $=$ Physiology and Chronic Health disease Classification System .

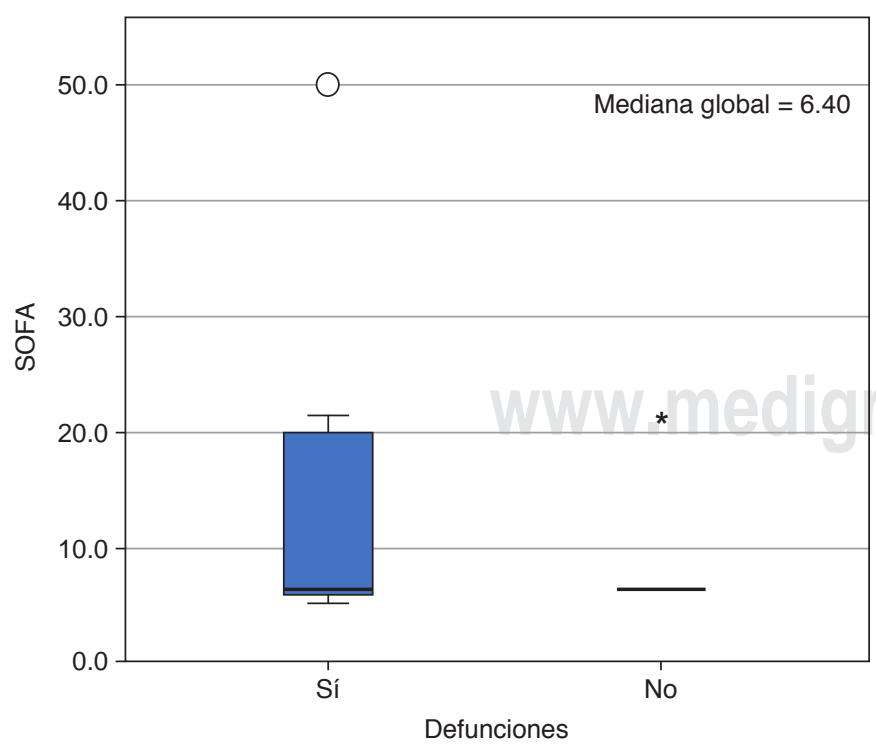

Figura 1: Diferencia de puntuación SOFA entre los grupos con fracaso de las puntas nasales de alto flujo y $\sin$ fracaso.

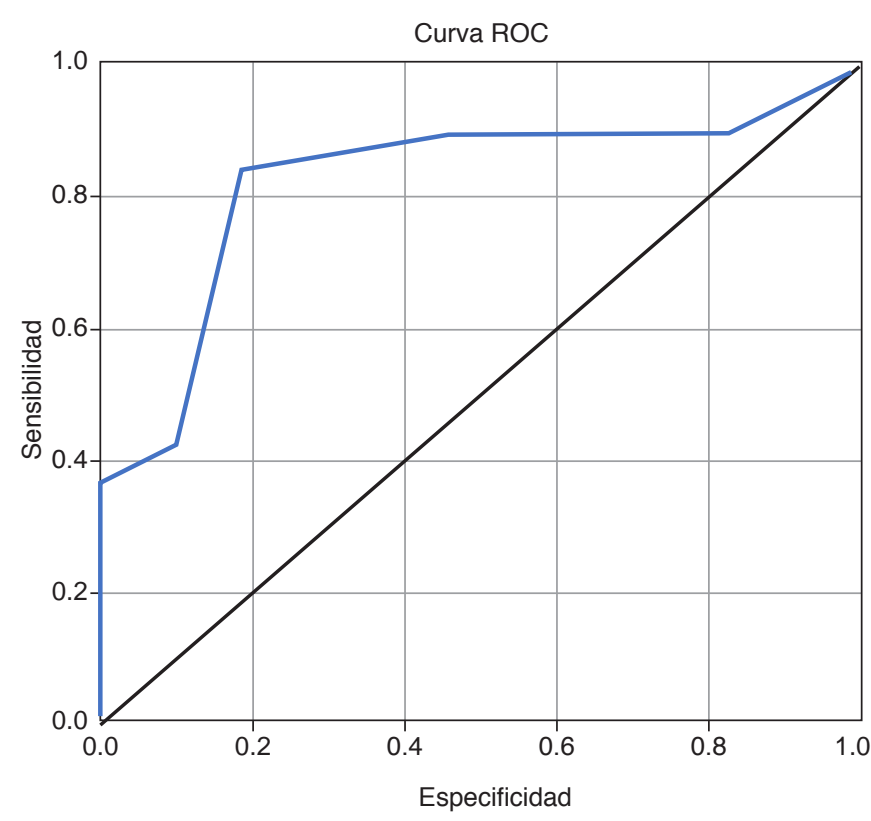

Figura 2: Curva ROC con puntaje $\geq 5$ puntos con área bajo la curva: $0.82 \%$, intervalo de confianza $95 \%(0.67-0.98)$, sensibilidad $84 \%$ y especificidad $81 \%$.

Tabla 4: Diferencias de las variables de la escala HACOR entre el grupo de fracaso y éxito de las puntas nasales de alto flujo.

\begin{tabular}{lccc}
\hline & $\begin{array}{c}\text { Con fracaso de } \\
\text { PNAF }\end{array}$ & Éxito de PNAF & $p$ \\
\hline $\mathrm{FC}$ & $85(60-110)$ & $80(49-117)$ & 0.168 \\
$\mathrm{FR}$ & $24(17-54)$ & $22.5(15-36)$ & 0.036 \\
$\mathrm{Glasgow}$ & $15(14-15)$ & $15(7-15)$ & 0.033 \\
$\mathrm{pH}$ & $7.40(7.30-7.50)$ & $7.45(7.20-7.50)$ & 0.317 \\
$\mathrm{SpO}_{2} / \mathrm{FiO}_{2}$ & $145(81-408)$ & $150(75-333)$ & 0.632 \\
\hline
\end{tabular}

$\mathrm{FC}=$ frecuencia cardiaca; $\mathrm{FR}$ = frecuencia respiratoria; $\mathrm{SpO}_{2}=$ saturación capilar de oxígeno; $\mathrm{FiO}_{2}$ = fracción inspirada de oxígeno.

(pacientes con una $\mathrm{PaO}_{2} / \mathrm{FiO}_{2}<200$ ) que la tasa de intubación era menor en los casos tratados con PNAF en comparación con los tratados con VNI u oxígeno estándar. ${ }^{15}$ En la actualidad, con la pandemia de SARSCoV-2 en nuestra UTI, las PNAF se han utilizado como primera medida de soporte respiratorio con resultados muy variables sin contar aún con una herramienta que pronostique de manera adecuada el fracaso de esta terapia y evitar el retraso de la intubación.

En aquéllos con insuficiencia respiratoria aguda, las PNAF suelen iniciarse en caso de persistencia de hipoxemia y/o dificultad respiratoria a pesar de la oxigenoterapia convencional. Por tanto, los niveles de hipoxemia de estos casos varían considerablemente, desde hipoxemia leve a moderada o grave $\left(\mathrm{PaO}_{2} /\right.$ $\mathrm{FiO}_{2}<200$ ). Aunque una proporción notable de ellos se trata con éxito con PNAF, no se ha establecido el límite inferior en términos de $\mathrm{PaO}_{2} / \mathrm{FiO}_{2} \cdot{ }^{13}$ En nuestro 
estudio se consideraron como casos que fracasaron al tratamiento con PNAF aquéllos con $\mathrm{PaO}_{2} / \mathrm{FiO}_{2}$ menor a $150 \mathrm{mmHg}$ de manera persistente y se decidió realizar una modificación mínima al índice $\mathrm{PaO}_{2} / \mathrm{FiO}_{2}$ por $\mathrm{SpO}_{2} / \mathrm{FiO}_{2}$ similar a un estudio publicado previamente por Contreras y colaboradores, donde obtuvieron una sensibilidad y especificidad con la escala HACOR similar a la obtenida por nosotros; sin embargo, este estudio fue realizado con ventilación mecánica no invasiva (VMNI) en una población heterogénea para facilitar la aplicación de la escala, ${ }^{16}$ además observaron mayor porcentaje de fracaso en el género masculino en los pacientes que tuvieron PNAF. Se ha documentado en varias publicaciones previas que el género masculino es un factor de riesgo para enfermedad más grave, peor pronóstico y mortalidad. ${ }^{17}$ Se ha planteado la hipótesis de que esto puede deberse a la presencia de andrógenos, o un nivel más bajo de anticuerpos del SARS-CoV-2 en comparación con las mujeres. ${ }^{18,19}$ Se encontró la presencia de niveles disminuidos de lipoproteínas de alta densidad (HDL, por sus siglas en inglés) en nuestra muestra, lo cual se ha documentado en diversas observaciones clínicas que demuestran que los niveles circulantes de HDL disminuyen durante la fase aguda del estado inflamatorio y especialmente durante la sepsis. Los efectos benéficos de las lipoproteínas en la sepsis pueden estar relacionados con su capacidad para neutralizar aproximadamente $90 \%$ de las sustancias bacterianas tóxicas (incluidos los lipopolisacáridos [LPS] y el ácido lipoteicoico). De todas las lipoproteínas, las HDL tiene la mayor capacidad de unión a LPS y ácido lipoteicoico. ${ }^{20-23}$

Con respecto a la mortalidad, todos los casos que presentaron falla a la ventilación con PNAF a las dos horas, la tasa de mortalidad fue de $100 \%$. La escala HACOR se ha validado en pacientes con ventilación mecánica no invasiva (VMNI) en poblaciones heterogéneas, principalmente EPOC e insuficiencia cardiaca; ${ }^{24,25}$ sin embargo, cuenta con variables clínicas fáciles de obtener que se pueden evaluar en casos con IRHA de origen infeccioso.

Algunos autores han publicado que los esfuerzos inspiratorios espontáneos vigorosos pueden conducir rápidamente a una lesión pulmonar autoinducida por el paciente (LPAP), por lo que la ventilación mecánica debe emplearse de manera temprana para evitar la progresión de una lesión leve a una lesión pulmonar más grave. ${ }^{26}$ La ventilación mecánica invasiva salva la vida en casos de insuficiencia respiratoria grave y pocas terapias médicas igualan su poder; sin embargo, la ventilación mecánica invasiva expone a los pacientes a complicaciones y aumenta la mortalidad, aún no es posible elegir un punto de corte de saturación de oxígeno en el que los beneficios de la ventilación mecánica superen decididamente sus peligros en todos los pa- cientes, por lo que sugieren retrasar la intubación. ${ }^{27}$ Es Importante disponer de una herramienta fácil de obtener que nos indique la necesidad de manejo avanzado de la vía aérea y no retrasar este proceso, en el cual se ha demostrado ampliamente el incremento de la mortalidad que representa.

\section{CONCLUSIONES}

La aplicación de la escala HACOR es un buen predictor de intubación y fracaso de las puntas nasales de alto flujo.

El uso de la relación $\mathrm{SpO}_{2} / \mathrm{FiO}_{2}$, el cual se sustituyó en este estudio, simplifica la aplicación y el monitoreo de pacientes con neumonía por COVID-19.

Un puntaje mayor o igual a 5 puntos en la escala HACOR predice fracaso de las PNAF y mayor mortalidad con una sensibilidad y especificidad mayor de $80 \%$ en los casos con neumonía por COVID-19.

El rendimiento del puntaje HACOR es similar si no se dispone de $\mathrm{pH}$ de la gasometría, por lo que su aplicación en un entorno de bajos recursos que no cuente con gasometría simplifica su utilización y puede aplicarse en los servicios de urgencias y hospitalización. Es el primer estudio que valora la escala HACOR en los pacientes con PNAF. La limitación de este estudio es el tamaño pequeño de la muestra y el haber sido efectuado en un solo centro hospitalario.

\section{AGRADECIMIENTOS}

A todo el personal que labora en la Unidad de Terapia Intensiva, Hospital Ángeles Mocel.

\section{REFERENCIAS}

1. Spoletini G, Alotaibi M, Blasi F, Hill NS. Heated humidified high flow nasal oxygen in adults. Mechanism of action and clinical implications. Chest. 2015;148(1):253-261.

2. Nava $\mathrm{S}$, Hill $\mathrm{N}$. Non invasive ventilation in acute respiratory failure. Lancet. 2009;374(9685):250-259.

3. Guan WJ, Ni Z, Hu Y, et al. Clinical characteristics of coronavirus disease 2019 in China. N Engl J Med. 2020;382:1708-1720.

4. Bazuaye EA, Stone TN, Corris PA, Gibson GJ. Variability of inspired oxygen concentration with nasal cannulas. Thorax. 1992;47(8):609-611.

5. Chanques G, Constantin JM, Sauter M, et al. Discomfort associated with under humidified high-flow oxygen therapy in critically ill patients. Intensive Care Med. 2009;35(6):996-1003.

6. Mauri T, Wang YM, Corte FD, et al. Nasal high flow: physiology, efficacy and safety in the acute care setting, a narrative review. Open Access Emerg Med. 2019;11:109-120.

7. Helviz Y, Einav S. A systematic review of the high-flow nasal cannula for adult patients. Crit Care. 2018;22(1):71.

8. Roca O, Riera J, Torres F, Masclan JR. High-flow oxygen therapy in acute respiratory failure. Respir Care. 2010;55(4):408-413.

9. Slutsky AS, Ranieri VM. Ventilator-induced lung injury. N Engl J Med. 2013;369(22):2126-2136.

10. Kalanuria AA, Ziai W, Mirski M. Ventilator-associated pneumonia in the ICU. Crit Care. 2014;18(2):208.

11. Kang BJ, Koh Y, Lim CM, et al. Failure of high-flow nasal cannula therapy may delay intubation and increase mortality. Intensive Care Med. 2015;41:623-632. 
12. Duan J, Han X, Bai L, Zhou L, Huang S. Assessment of heart rate, acidosis, consciousness, oxygenation, and respiratory rate to predict noninvasive ventilation failure in hypoxemic patients. Intensive Care Med. 2017;43(2):192-199.

13. Ricard JD, Roca O, Lemiale V, et al. Use of nasal high flow oxygen during acute respiratory failure. Intensive Care Med. 2020;46:2238-2247.

14. Sztrymf B, Messika J, Bertrand F, et al. Beneficial effects of humidified high flow nasal oxygen in critical care patients: a prospective pilot study. Intensive Care Med. 2011;37(11):17801786.

15. Frat JP, Thille AW, Mercat A, et al. High-flow oxygen through nasal cannula in acute hypoxemic respiratory failure. $N$ Engl J Med. 2015;372(23):2185-2196.

16. Contreras A, Varela LE, Gaytán CJ, et al. Utilidad de escala HACOR para predecir falla de la ventilación mecánica no invasiva y mortalidad en las Unidades de Terapia Intensiva del Centro Médico ABC. An Med (Mex). 2018;63(4):261-265.

17. De Lusignan S, Dorward J, Correa A, et al. Risk factors for SARS-CoV-2 among patients in the Oxford Royal College of General Practitioners Research and Surveillance Centre primary care network: a cross-sectional study. Lancet Infect Dis. 2020;20(9):1034-1042.

18. Montopoli M, Zumerle S, Vettor R, et al. Androgen-deprivation therapies for prostate cancer and risk of infection by SARSCoV-2: a population-based study $(n=4532)$. Ann Oncol. 2020;31(8):1040-1050.

19. Zeng F, Dai C, Cai P, et al. A comparison study of SARS-CoV-2 IgG antibody between male and female COVID-19 patients: a possible reason underlying different outcome between sex. $J$ Med Virol. 2020;92(10):2050-2054.
20. Van Leeuwen HJ, Heezius EC, Dallinga GM, Van Strijp JA, Verhoef J, Van Kessel KP. Lipoprotein metabolism in patients with severe sepsis. Crit Care Med. 2003;31(5):1359-1366.

21. Barlage S, Gnewuch C, Liebisch G, et al. Changes in HDLassociated apolipoproteins relate to mortality in human sepsis and correlate to monocyte and platelet activation. Intensive Care Med. 2009;35(11):1877-1885.

22. Chien JY, Jerng JS, Yu CJ, Yang PC. Low serum level of highdensity lipoprotein cholesterol is a poor prognostic factor for severe sepsis. Crit Care Med. 2005;33(8):1688-1693.

23. Cirstea M, Walley KR, Russell JA, Brunham LR, Genga KR, Boyd JH. Decreased high-density lipoprotein cholesterol level is an early prognostic marker for organ dysfunction and death in patients with suspected sepsis. J Crit Care. 2017;38:289-294.

24. Duan J, Wang S, Liu P, et al. Early prediction of noninvasive ventilation failure in COPD patients: derivation, internal validation, and external validation of a simple risk score. Ann Intensive Care. 2019;9(1):108.

25. Carrillo A, Lopez A, Carrillo L. Validity of a clinical scale in predicting the failure of non-invasive ventilation in hypoxemic patients. J Crit Care. 2020;60:152-158.

26. Marini JJ, Gattinoni L. Management of COVID-19 respiratory distress. JAMA. 2020;323(22):2329-2330.

27. Tobin MJ, Laghi F, Jubran A. P-SILI is not justification for intubation of COVID-19 patients. Ann Intensive Care. 2020;10:105.

\section{Correspondencia:}

Ibzan Jahzeel Salvador-Ibarra

E-mail: ibzjah@gmail.com 